\title{
Zoledronic acid-induced symmetrical drug-related intertriginous and flexural exanthema (SDRIFE)
}

\author{
Hyndavi Kandala, ${ }^{1}$ Luis Fernando Gonzalez-Mosquera, ${ }^{1}$ Priyanka Barua, ${ }^{2}$ \\ Alida Podrumar ${ }^{2}$
}

${ }^{1}$ Internal Medicine, Nassau University Medical Center, East Meadow, New York, USA ${ }^{2}$ Medicine-heme/onc, Nassau University Medical Center, East Meadow, New York, USA

\section{Correspondence to}

Dr Hyndavi Kandala; hyndavikandala@gmail.com

Accepted 2 September 2021

\section{DESCRIPTION}

The patient is a 60-year-old female with invasive ductal carcinoma of left breast, Estrogen Receptor/ Progesterone Receptor positive, Human Epidermal Growth factor 2 (HER2) negative, status post left mastectomy and chemoradiation in 2014. The patient is currently being treated for metastatic disease with palbociclib + fulvestrant and was given zoledronic acid (Zometa) for bone metastasis.

The day after zoledronic acid infusion, the patient complained of a red itchy rash in the axillary and inguinal regions, associated with diffuse myalgias. She denied shortness of breath, hoarseness, tongue swelling, abdominal pain, nausea and vomiting. Her vitals were stable. Physical examination demonstrated a diffuse non-tender erythematous rash near the axillary (figure 1) and groin regions (figure 2),

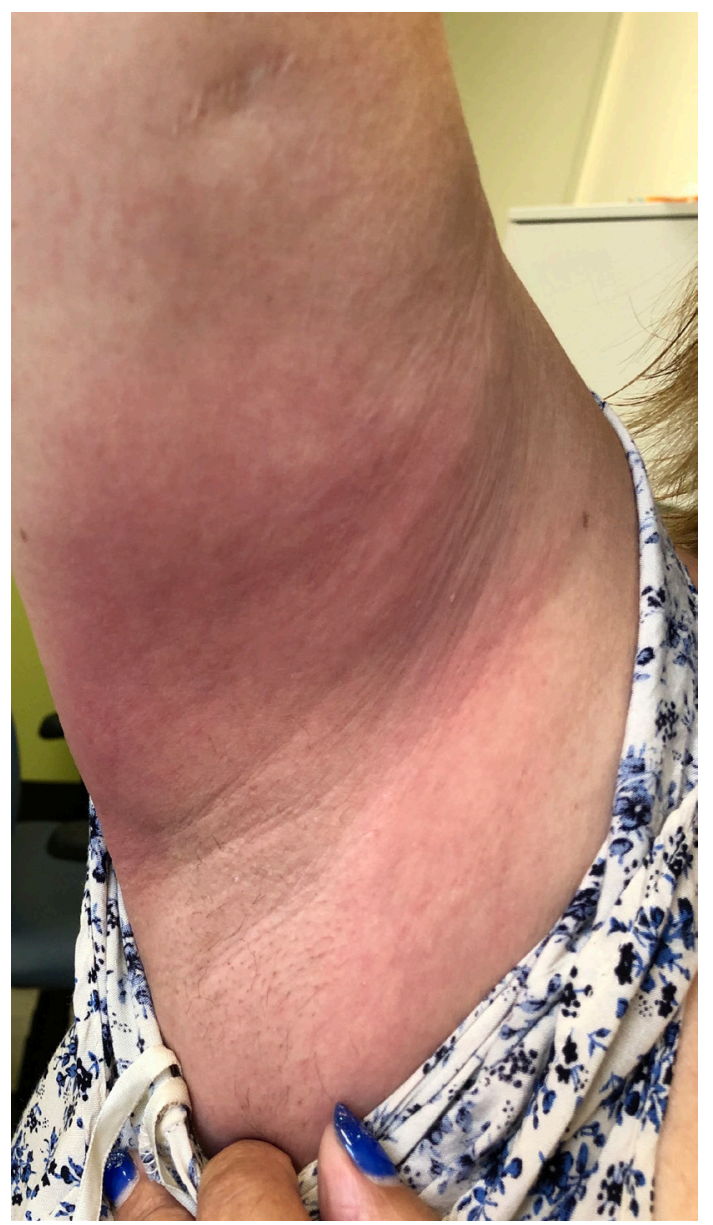

Figure 1 Erythematous rash in the axillary region with no papules or vesicles.

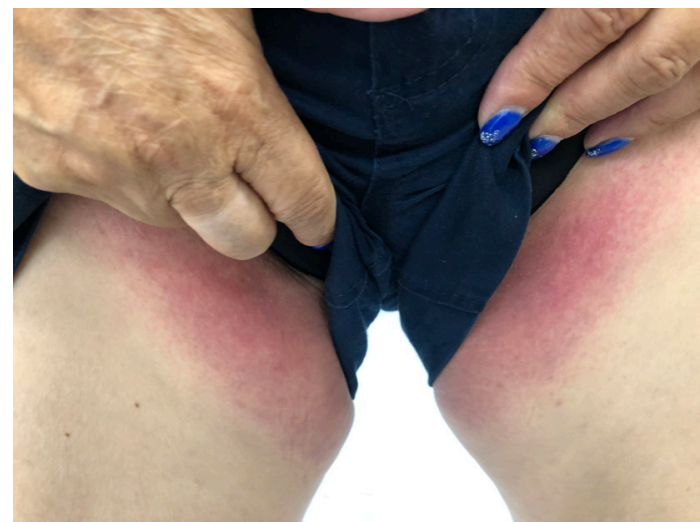

Figure 2 Symmetric erythematous rash located in the groin area.

with regression in the periphery of lesions. No papules, vesicles or discharge were noted.

Symmetrical drug-related intertriginous and flexural exanthema (SDRIFE) is a rare cutaneous reaction observed after exposure to systemic medications, ${ }^{1}$ more commonly with beta-lactam antibiotics; other medications associated are pseudoephedrine, codeine, cimetidine, nystatin and fluconazole. ${ }^{2}$ Literature regarding this is limited. Since 1984, only about a hundred cases have been reported so far, and only one among them was related to zoledronic acid. ${ }^{34}$ The diagnostic criteria proposed in 2004 for SDRIFE are shown in box $1 .^{5}$ The exact mechanism of SDRIFE is unknown; however, some authors believe that it is due to a T-cell-mediated type IV delayed hypersensitivity. ${ }^{12}$

The differentials for a rash are extensive. Recurring skin lesions in the same location would make fixed drug eruptions a possibility but can be ruled out with a complete blood count and comprehensive metabolic panel. ${ }^{6}$ Acute generalised exanthematous pustulosis will have the characteristic pustular eruptions. Baboon syndrome may also present with a similar rash from exposure to both contact allergens and systemic drugs, ${ }^{7}$ whereas SDRIFE is mainly due to systemic drugs exposure. Other rashes such as allergic contact dermatitis, intertrigo and seborrheic dermatitis can easily be distinguished from history.

The diagnosis of SDRIFE is mainly based on clinical presentation and exclusion of other causes of a similar rash. ${ }^{7}$ A patch test gives a positive reaction in only up to $50 \%$ of patients with SDRIFE. Controlled drug-provocation testing is the gold standard clinical test and gives a positive result in most patients with SDRIFE. ${ }^{7}$ Our patient presented 
with symmetrical involvement of more than one intertriginous area without systemic symptoms, which began after zoledronic acid infusion, meeting the clinical criteria of SDRIFE. Treatment is usually conservative measures, including steroids and antihistamines, for the symptoms. Lesions typically disappear

Box 1 Diagnostic criteria for systemic drug-related intertriginous and flexural exanthema syndrome

1. Exposure to a systemically administered drug either at the first or a repeated dose.

2. Sharply demarcated erythema of the gluteal/perianal area and/or V-shaped erythema of the inguinal/perigenital area.

3. Involvement of at least one other intertriginous/flexural localisation.

4. The symmetry of affected areas.

5. Absence of systemic symptoms and signs.

\section{Learning points}

- Erythematous rash in intertriginous and flexural regions after administration of systemic drugs should raise the concern of symmetrical drug-related intertriginous and flexural exanthema (SDRIFE).

- Out of the systemic drugs recognised to cause SDRIFE, zoledronic acid should be considered as one of the possible causative drugs.

- Treatment is symptomatic with conservative measures and withdrawal of the offensive agent. after withdrawal of the causative drug. ${ }^{8}$ In our patient, the rash resolved in a few days without any significant intervention.

Contributors The authors HK and LFG-M have contributed equally to the case report. PB provided the pictures and edited the document. AP reviewed the article and provided feedback which was used to update and finalise the manuscript.

Funding The authors have not declared a specific grant for this research from any funding agency in the public, commercial or not-for-profit sectors.

Competing interests None declared.

Patient consent for publication Obtained

Provenance and peer review Not commissioned; externally peer reviewed.

\section{REFERENCES}

1 Huynh T, Hughey LC, McKay K, et al. Systemic drug-related intertriginous and flexural exanthema from radio contrast media: A series of 3 cases. JAAD Case Rep 2015;1:147-9.

2 Mohapatra M, Panda M, Kar BR, et al. Symmetric drug-related Intertriginous and flexural Exanthema due to itraconazole: an uncommon side effect of a commonly used drug. Indian Dermatol Online J 2017;8:501-3.

3 Cohen PR. Zoledronic acid-associated symmetrical drug-related intertriginous and flexural exanthema (SDRIFE): report of baboon syndrome in a woman with recurrent metastatic breast cancer after receiving zoledronic acid. Dermatol Online J 2015;21.

4 Harbaoui S, Litaiem N. Symmetrical drug-related Intertriginous and flexural Exanthema. Treasure Island (FL): StatPearls Publishing, 2021. https://www.ncbi.n/m.nih.gov/books/ NBK539750/

5 Häusermann P, Harr T, Bircher AJ. Baboon syndrome resulting from systemic drugs: is there strife between SDRIFE and allergic contact dermatitis syndrome? Contact Dermatitis 2004;51:297-310.

6 McNamara K, Hughes OB, Strowd LC. Cutaneous drug eruptions including serum sickness-like reaction, symmetrical drug-related intertriginous and flexural exanthema, and drug-induced lupus. Clin Dermatol 2020;38:641-7.

7 Nespoulous L, Matei I, Charissoux A, et al. Symmetrical drug-related intertriginous and flexural exanthema (SDRIFE) associated with pristinamycin, secnidazole, and nefopam, with a review of the literature. Contact Dermatitis 2018;79:378-80.

8 Weiss D, Kinaciyan T. Symmetrical drug-related intertriginous and flexural exanthema (SDRIFE) induced by mefenamic acid. JAAD Case Rep 2019;5:89-90.

Copyright 2021 BMJ Publishing Group. All rights reserved. For permission to reuse any of this content visit

https://www.bmj.com/company/products-services/rights-and-licensing/permissions/

BMJ Case Report Fellows may re-use this article for personal use and teaching without any further permission.

Become a Fellow of BMJ Case Reports today and you can:

- Submit as many cases as you like

- Enjoy fast sympathetic peer review and rapid publication of accepted articles

- Access all the published articles

- Re-use any of the published material for personal use and teaching without further permission

Customer Service

If you have any further queries about your subscription, please contact our customer services team on +44 (0) 2071111105 or via email at support@bmj.com.

Visit casereports.bmj.com for more articles like this and to become a Fellow 